https://helda.helsinki.fi

Canine mammary tumour cells exposure to sevoflurane : effects on cell proliferation and neuroepithelial transforming gene 1 expression

\title{
Argano, Martina
}

2019-05

Argano, M , De Maria , R , Vogl , C , Rodlsberger , K, Buracco , P \& Menzies, M P L 2019 ,

' Canine mammary tumour cells exposure to sevoflurane : effects on cell proliferation and neuroepithelial transforming gene 1 expression ' , Veterinary Anaesthesia and Analgesia , vol. 46 , no. 3 , pp. 369-374 . https://doi.org/10.1016/j.vaa.2018.12.006

http://hdl.handle.net/10138/314689

https://doi.org/10.1016/j.vaa.2018.12.006

cc_by_nc_nd

acceptedVersion

Downloaded from Helda, University of Helsinki institutional repository.

This is an electronic reprint of the original article.

This reprint may differ from the original in pagination and typographic detail.

Please cite the original version. 


\section{SHORT COMMUNICATION}

\section{Canine mammary tumour cells exposure to sevoflurane: effects on proliferation and neuroepithelial transforming gene 1 expression.}

\section{Abstract}

Objective The influence of perioperative factors, such as anaesthetic and analgesic techniques, on metastatic spread following surgery for primary cancer removal is of growing interest. The present study investigated the effects of sevoflurane on canine mammary tumour cell proliferation (MTT colorimetric assay) and on the expression of the neuroepithelial transforming gene 1 (NET1).

Study design Prospective controlled in vitro trial.

Study material Primary canine tubular adenocarcinoma (CIPp) and metastatic canine tubular adenocarcinoma (CIPm) cells.

Methods To perform the MTT tests, cell lines were seeded at a density of 3,000 cells per well and incubated with sevoflurane $(1,2.5$ or $4 \mathrm{mM})$ or only with the culture medium (control). Sevoflurane was added to the cell cultures every hour to avoid changes in drug concentration. MTT assays were performed after 6 hours of exposure obtaining absolute values of absorbance. The RNA isolated from the lysates of the same cell lines underwent quantitative polymerase chain reaction to evaluate NET1 gene expression changes compared to controls. One- and 2-way ANOVAs were used as appropriate $(p<0.05)$.

Results A significant increase in cell proliferation compared to controls was observed in CIPp treated with lower sevoflurane concentrations, while a significant decrease in cell proliferation was found in CIPm treated with all the sevoflurane concentrations. All treatments of CIPp did not induce changes in gene expression compared to controls, while a significant increase in gene expression was observed in CIPm between controls and the higher sevoflurane concentration.

Conclusions and clinical relevance Sevoflurane treatments modified the cell proliferation rate in both cell lines showing an increase or a decrease when applied to primary or metastatic canine tubular adenocarcinoma cells, respectively. Expression of the NET1 gene increased after treatment 
with sevoflurane $4 \mathrm{mM}$ in metastatic cells. The role of sevoflurane on cancer recurrence should be further investigated.

Keywords canine mammary tumour cells, cell proliferation, MTT assay, NET1 gene, tumour, sevoflurane

\section{Introduction}

Malignant mammary tumours are a significant cause of morbidity and mortality in dogs, representing one of the most common types of cancer and causes of cancer-related death (Karayannopoulou \& Lafioniatis 2016), even though the incidence of severe canine mammary tumour cases has been reduced in regions that regularly perform early sterilization (Vascellari et al. 2016). Usually, death is the result of recurrence and metastasis (Vascellari et al. 2016). Available treatments are numerous, however, surgical removal of the primary mass is still a major pillar (Karayannopoulou \& Lafioniatis 2016). Therefore, the possible influence of perioperative factors on metastatic spread, such as anaesthetic and analgesic techniques, is of growing interest and has been investigated by recent retrospective studies in human medicine (Wigmore et al. 2016).

In vitro (Ecimovic et al. 2013) and retrospective clinical trials (Wigmore et al. 2016) have shown that volatile agents like sevoflurane might have a pro-tumourigenic effect and consequently facilitate the development of metastasis in many solid tumours (Wigmore et al. 2016). In particular, sevoflurane has been shown to increase human breast cancer cell proliferation, migration and invasion in vitro (Ecimovic et al. 2013).

Volatile agents are commonly used to maintain anaesthesia of dogs undergoing mastectomy. It is unknown whether sevoflurane could potentially facilitate tumour cell proliferation and migration in this species. Therefore, this study was designed to evaluate, in vitro, sevoflurane's ability to affect primary and metastatic canine mammary tumour cell proliferation using a colorimetric assay (MTT: 3-(4,5-dimethylthiazol-2-yl)-2,5-diphenyl tetrasodium bromide). In addition, sevoflurane's effects on the expression of neuroepithelial transforming gene 1 (NET1), that has been associated with cell migration ability (Ecimovic et al. 2014), was also investigated. We hypothesized that 6 hours of 
sevoflurane exposure to canine mammary tumour cells would lead to an increase in cell proliferation and NET1 gene expression.

\section{Materials and methods}

Cell culture

Established canine mammary tubular adenocarcinoma cell lines derived from one individual's primary (CIPp) and metastatic (CIPm) lesions were used (Uyama et al. 2006). Cells were grown in Roswell Park Memorial Institute medium supplemented with 10\% foetal bovine serum (SigmaAldrich, MO, USA), $100 \mu \mathrm{g} \mathrm{mL} \mathrm{m}^{-1}$ penicillin (Sigma-Aldrich), $100 \mu \mathrm{g} \mathrm{mL} \mathrm{m}^{-1}$ streptomycin (SigmaAldrich), $1.5 \mathrm{mg} \mathrm{mL}^{-1}$ amphotericin B (Sigma-Aldrich) and incubated for 24 hours at $37^{\circ} \mathrm{C}$ in a humidified atmosphere with 5\% carbon dioxide.

\section{MTT assay}

Cells were grown in $75 \mathrm{~cm}^{2}$ standard tissue culture flask (Sarstedt Ltd, Ireland) as monolayers. Media were changed every three days. Before each experiment, cells were harvested from $70 \%$ confluent cultures by trypsinization and counted with an automated cell counter (Automated Cell Counter TC20; Bio-Rad, Italy). Cells were seeded at a density of 3,000 cells per well for a total of six experimental wells in a 96-well cell culture plate (Eppendorf Cell Culture Plate, Eppendorf S.r.l., Italy). The number of 3,000 cells per well was chosen from the preliminary evaluation of a time-dependent exponential cell growth curve. Cells were seeded in triplicates in a concentration range from 1,000 to 10,000 cells per well and incubated for 4, 6 and 12 hours. Subsequently, the proliferation index was assessed using the MTT colorimetric assay. The concentration of 3,000 cells per well was found optimal to show time related cell growth. Before treatment exposure, cells were incubated for 12 hours with $100 \mu \mathrm{L}$ of normal culture medium to allow homogeneous cell adhesion. A clinically available sevoflurane formulation (Sevorane; AbbVie Oy, Finland) was utilized for the treatment in three different concentrations: $1,2.5$ or $4 \mathrm{mM}$ (treatments: S1, S2.5 and S4, 
respectively). Cells grown only in the culture medium were used as control. Medium containing sevoflurane (S1, S2.5 and S4 concentrations) was added every hour to the culture medium to avoid decreases in drug concentration over time due to evaporation (Ecimovic et al. 2013). Treatments were removed after 6 hours and cell survival and proliferation assessed with an MTT colorimetric assay according to Tada et al. (1986). Briefly, $20 \mu \mathrm{L}$ of MTT were diluted in phosphate-buffered saline to reach a concentration of $5 \mathrm{mg} \mathrm{mL}^{-1}$ and a $\mathrm{pH}$ of 7.5 . The solution was added to each well and incubated for 4 hours at $37^{\circ} \mathrm{C}$. Subsequently, $0.1 \mathrm{~mL}$ of $10 \%$ sodium dodecyl sulfate (SigmaAldrich) diluted in a solution of $0.01 \mathrm{M} \mathrm{HCl}$ was added to each well and incubated overnight. Absolute absorbance was then measured with a spectrophotometer (Microplate Model 680, Bio$\mathrm{Rad}$ ) on an ELISA plate reader with a wavelength of $590 \mathrm{~nm}$. Values lower and higher than controls indicated reduction and increase in cell proliferation after treatment, respectively.

Quantitative PCR

Three hundred thousand cells were seeded in triplicates in p6 culture plates (Eppendorf Cell Culture Plate; Eppendorf S.r.1.). After 6 hours of incubation to permit cell attachment, they were treated with 1 or $4 \mathrm{mM}$ of sevoflurane (treatments: S1 and S4, respectively) for 6 hours. Similarly to the MTT assay and to compensate for the evaporation tendency of this agent, the same concentrations of sevoflurane were added to the cell cultures every hour. Cells cultured without treatment were used as controls. In order to isolate total ribonucleic acid (RNA), culture media were removed at the end of the treatment and $0.5 \mathrm{~mL}$ of a ready-to-use reagent designed to isolate high quality total RNA (TRIzol, Sigma-Aldrich, Dublin, Ireland) was added to each well to lyse the cells, according to manufacturer`s instructions. Once a microscopic examination revealed cells to be lysed, the cell lysate was transferred to a $1.5 \mathrm{~mL}$ microfuge tube. Thereafter, $200 \mu \mathrm{L}$ of chloroform were added. The mixture was gently shaken, left at room temperature $\left(25^{\circ} \mathrm{C}\right)$ for 15 minutes and centrifuged at 13,000 revolutions per minute $(\mathrm{RPM})$ for 15 minutes at $4^{\circ} \mathrm{C}$. The upper aqueous layer was transposed into another $1.5 \mathrm{~mL}$ tube carefully without touching the genomic and protein-containing interphase. A total of $0.5 \mathrm{~mL}$ of ice-cold isopropanol was added to the aqueous phase, the tube 
gently shaken and left to stand on ice for 10 minutes before being centrifuged at 13,000 RPM for another 10 minutes at $4^{\circ} \mathrm{C}$. The supernatant was removed and $1 \mathrm{~mL}$ of sterile ethanol $(75 \%)$ was added to wash the pellet by gently centrifuging (7,500 RPM for 5 minutes). After ethanol removal, the pellet was let to air-dry for 5 minutes before being re-suspended in $50 \mu \mathrm{L}$ of nuclease-free water by heating it at $60^{\circ} \mathrm{C}$ for 15 minutes. Total RNA was quantified with an automated electrophoresis system (Experion Electrophoresis System; Bio-Rad,) and complementary deoxyribonucleic acid (cDNA) was synthesized from $1 \mu \mathrm{g}$ of total RNA using a reverse transcription kit (QuantiTect Reverse Transcription kit; Qiagen, Italy). According to manufacturer's instructions, $1 \mu \mathrm{g}$ of total RNA was incubated with $2 \mu \mathrm{L}$ of DNAse buffer treatment (gDNA Wipeout Buffer; Qiagen,) and RNAse free water to reach a total volume of $14 \mu \mathrm{L}$ for 2 minutes at $42^{\circ} \mathrm{C}$ and left for 10 minutes on ice. Thereafter, $1 \mu \mathrm{L}$ of reverse transcriptase (Quantiscript Reverse Transcriptase; Qiagen), $4 \mu \mathrm{L}$ of a dedicated buffer (Quantiscript RT Buffer 5X; Qiagen,) and $1 \mu \mathrm{L}$ of a dedicated primer mix (RT Primer mix; Qiagen,) were added and incubated for 15 minutes at $42^{\circ} \mathrm{C}$ following 3 minutes at $95^{\circ} \mathrm{C}$ to inactivate the reverse transcriptase. One $\mu \mathrm{L}$ of cDNA was used for quantitative polymerase chain reaction (qPCR) to evaluate the relative amount of specific NET1 gene transcript. One $\mu \mathrm{L}$ of cDNA was subjected to qPCR with a dedicated detection chemistry system (IQ SYBR Green Supermix; Bio-Rad) and an optical software system (IQ5 Optical System Software; Bio-Rad,). The sequences of primers used were: canine glyceraldehyde-3-phosphate dehydrogenase (GAPDH, gene bank entry AB038240.1) forward 5'-GGCACAGTCAAGGCTGAGAAC-3', canine GAPDH reverse 5'-CCAGCATCACCCCATTTGAT-3', canine NET1 (Gene bank entry XM_54427.5) forward 5'-CATCAAGAGGACGATCCGGG-3', and canine NET1 reverse 5'ATTGCTTGGCTCCTCTTGCT-3'. The reaction conditions were: reverse transcription, 3 minutes at $95^{\circ} \mathrm{C}\left(1\right.$ cycle) followed by denaturation for 30 seconds at $95^{\circ} \mathrm{C}$ and annealing for 30 seconds at $60^{\circ} \mathrm{C}$ (35 cycles). Glyceraldehyde-3-phosphate dehydrogenase expression levels were used to normalize NET1 gene expression. Gene expression was calculated using a relative quantification assay corresponding to the comparative cycle threshold $(\mathrm{Ct})$ method: the amount of target gene, 
normalized to the endogenous housekeeping gene (GAPDH) and relative to the calibrator (control sample), was then transformed by $2 \Delta \Delta \mathrm{Ct}$ (one fold increase), where $\Delta \Delta \mathrm{Ct}=\Delta \mathrm{Ct}$ (sample) $-\Delta \mathrm{Ct}$ (control) and $\Delta \mathrm{Ct}$ is the $\mathrm{Ct}$ of the target gene subtracted from the $\mathrm{Ct}$ of the housekeeping gene. Values for $\Delta \mathrm{Ct}$ were obtained in triplicate for each sample.

132 Statistical analysis

133 One overall mean value of $\Delta \mathrm{Ct}$ was used for statistical analysis per biological sample (i.e. mean 134 values of the technical triplicates). After log-transformation, residuals were approximately normal and variances approximately equal in all groups (visual inspection). One- and 2-way ANOVA tests were used to analyze the data. In the 2-way ANOVA test, the target variables were the mean values of absorbance at 6 hours of treatment, while the treatment (the three sevoflurane concentrations and the control) and the cell-type (primary and metastatic cells) were used as explanatory variables. In the one-way ANOVAs, the target variables were mRNA expressions of the NET1 gene and the treatment (two sevoflurane concentrations, S1 and S4, and the control) were the explanatory variables. In both sets of ANOVAs, pairwise differences between treatments and controls were tested for significance; a value of $p<0.05$ was considered statistically significant. All statistical analyses were performed with an open-source statistical software package (R-studio, version 3.2.0; www.r-project.org, MA, USA). Data are presented as mean \pm standard error and ranges for the $\%$ increases or decreases of cell proliferation rate.

\section{Results}

148 Mean \pm standard errors absorbance values are displayed in Figures 1a and 1b. A statistically 149 significant increase in cell proliferation rate compared to controls was observed in CIPp treated with S1 and S2.5 (Fig. 1a) of 23\% and 13\%, respectively. Conversely, a significant decrease in cell proliferation rate was found in CIPm treated with all the tested concentrations of sevoflurane (Fig. $1 \mathrm{~b} ; \mathrm{S} 1=-33 \%, \mathrm{~S} 2.5=-41 \%$ and $\mathrm{S} 4=-62 \%)$. 
Both, S1 and S4 did not induce any significant change in NET1 gene expression in CIPp cells compared to controls (Fig. 1c). A significant increase in gene expression was observed only in CIPm between controls and the cells treated with S4 (Fig. 1d).

\section{Discussion}

In the present study, a commercially available sevoflurane formulation effectively modified cellular proliferation in both cell lines in a divergent manner, increasing cell proliferation in CIPp but decreasing it in CIPm. Interestingly, NET1 gene expression was significantly increased only in CIPm cells treated with the higher concentration of sevoflurane.

Both tests, MTT and qPCR, have been extensively used in in-vitro cancer research (van Meerloo et al. 2011; Ecimovic et al. 2014). The MTT test is frequently used for the evaluation of the number of viable cells. The test measures the conversion of MTT into purple-coloured formazan crystals, which are induced by living cells' redox activity. A cellular redox activity decrease indicates reduced cell viability or decreased cell number while a cellular redox increase indicates cell viability or cell number increase. For the case of cancer cells, an increase or decrease in cell number count can be interpreted as an increase or decrease of the proliferation rate of the studied cells (van Meerloo et al. 2011). Therefore, in the current study, it may be inferred that sevoflurane prevents the proliferation of CIPm but enhances the proliferation of CIPp.

Present study findings are not completely in line with what is available in the scientific literature. Ecimovic and colleagues (2013) showed that 6 hours of sevoflurane exposure at the concentrations of 2, 3 and $4 \mathrm{mM}$ increased cell proliferation by $50-63 \%$ and by $50-67 \%$ in metastatic human breast adenocarcinoma cells that were oestrogen receptor positive (MCF7 cell line) or negative (MDA-MB-231 cell line), respectively. Controversially, a sevoflurane (2 mM) anti-proliferative effect was shown in C6 glioma cells (O’Leary et al. 2000). It should be noticed that, apart from being different tissue cells, the C6 glioma cells were not in a tumour transformation state, thus 
possibly reflecting the role of cell type and cell evolutional phase, rather than other factors like concentration and contact time, on the ability to respond to drug exposure.

To the authors' knowledge, this is the first study to investigate the effects of sevoflurane on NET1 gene expression. The NET1 gene is a RhoA specific guanine nucleotide exchange factor that enables tumour cells to invade and migrate (Ecimovic et al. 2014). The NET1 plays an important role in cytoskeletal reorganization, $\mathrm{N}$-cadherin expression and RhoA activation (Ecimovic et al. 2014). Therefore, an increased NET1 expression has been associated with malignant cellular behaviours (Leyden et al. 2006). Consistently, NET1 was described as being overexpressed in highly invasive cancer types such as human breast and gastric adenocarcinomas (Leyden et al. 2006). Expression of NET1 seems to be affected by some medications used in the perioperative period (Ecimovic et al. 2014). In the present study, NET1 expression was only increased in CIPm after being exposed to the higher evaluated concentration of sevoflurane (i.e. $4 \mathrm{mM}$ ). This could be interpreted as sevoflurane enhancing the migration ability of CIPm.

Exposure time and concentrations used in the present study were chosen based on what has been reported in human medicine, in order to make reasonable comparisons between studies (Ecimovic et al. 2013). Sevoflurane concentrations chosen by previous authors were made after evaluating sevoflurane plasma concentrations observed in people undergoing elective cardiac surgery and receiving sevoflurane $1.8 \%$ inspiratory volume. Considering that the sevoflurane minimal alveolar concentration in dogs is similar to that reported in people, it was assumed that concentrations between 1 and $4 \mathrm{mM}$ applied to the cell cultures would resemble the plasma concentrations of dogs anesthetized with sevoflurane in clinical practice.

The in vitro nature of the present study presents some limitations. Firstly, the mechanisms studied are only small pieces of the big puzzle of cancer propagation. Indeed, mechanisms that influence cancer recurrence are extremely numerous and complex and it cannot be excluded that sevoflurane influences cancer cells migration and proliferation by other means such as the modulation of the immune system or the up-regulation of hypoxia-inducible stress factors. In addition, the 
concomitant effects of other agents given in the routine clinical practice could potentially interfere

206 with sevoflurane effects on cancer cells. For instance, it was shown that serum from patients with 207 breast cancer who received general anaesthesia in the form of sevoflurane and systemic opioids 208 applied to MDA-MB-231 breast cancer cells increased both proliferation and migration of cancer 209 cells compared with serum of patients receiving propofol infusions and paravertebral blocks 210 (Deegan et al. 2009). Finally, it is difficult to extrapolate in vitro results to in vivo conditions. 211 Interestingly, a large retrospective study evaluating long-term survival of lung cancer patients 212 undergoing volatile or intravenous anaesthesia for elective surgery showed a statistically and 213 clinically significant survival time reduction in patients receiving inhalational anaesthetics 214 including sevoflurane (Wigmore et al. 2016).

215 In conclusion, sevoflurane treatments modified cell proliferation rate in both cell lines showing an 216 increase or a decrease when applied on CIPp or CIPm cells, respectively, compared to cell growth 217 in the sole cell culture medium. The expression of NET1 gene increased only after treatment with 218 sevoflurane $4 \mathrm{mM}$ in metastatic cells. Further studies are much needed for a better understanding of 219 the role of sevoflurane on canine mammary cancer cells. 


\section{References}

Deegan CA, Murray D, Doran P, et al (2009) Effect of anaesthetic technique on oestrogen receptornegative breast cancer cell function in vitro. Br J Anaesth 103, 685-690.

Ecimovic P, Mchugh B, Murray D et al (2013) Effects of Sevoflurane on Breast Cancer Cell Function In Vitro. Anticancer Res 33(10), 4255-4260.

Ecimovic P, Murray D, Doran PP, Buggy DJ (2014) Propofol and bupivacaine in breast cancer cell function in vitro - Role of the NET1 gene. Anticancer Res 34, 1321-1332.

Karayannopoulou M, Lafioniatis S (2016) Recent advances on canine mammary cancer of gastric cancer. Br J Cancer 94(8), 1204-1212.

O’Leary G, Bacon CL, Odumeru O, et al (2000) Antiproliferative actions of inhalational anesthetics: comparisons to the valproate teratogen. Int J Dev Neurosci 18(1), 39-45. 
247 Figure Legend

248 Figure 1 Changes in absorbance values in primary (a; CIPp) and metastatic (b; CIPm) canine 249 mammary tubular adenocarcinoma cells receiving different concentrations (S1: $1 \mathrm{mM}$; S2.5: 2.5 $250 \mathrm{mM}$; S4: $4 \mathrm{mM}$ ) of a commercially available sevoflurane formulation when compared to control 251 cells (C). Fold changes in NET1 gene expression in primary (c; CIPp) and metastatic (d; CIPm) 252 canine mammary tubular adenocarcinoma cells receiving different concentrations of a 253 commercially available sevoflurane formulation when compared to control cells $(* * *: p<0.001$; $254 * *: p<0.01 ; *: p<0.05)$ 